\title{
Crescimento de Aegla platensis Schmitt em ambiente natural (Crustacea, Decapoda, Aeglidae) ${ }^{1}$
}

\author{
Alessandra Angélica de Pádua Bueno ${ }^{2}$ \\ Georgina Bond-Buckup ${ }^{2}$ \\ Ludwig Buckup ${ }^{2}$
}

\begin{abstract}
The growth of Aegla platensis Schmitt (Crustacea, Decapoda, Aeglidae) in natural habitat. The crustaceans of the genus Aegla Leach, 1820 are found in streams, rivers and lakes from Franca, São Paulo, Brazil ( $\left.20^{\circ} 60^{\prime} \mathrm{S}, 47^{\circ} 40^{\prime} \mathrm{W}\right)$, to Ilha Madre de Dios, Chile $\left(50^{\circ} 01^{\prime} 10^{\prime \prime} \mathrm{S}, 75^{\circ} 18^{\prime} 45^{\prime \prime} \mathrm{W}\right)$ in South America. The growth and cheliped asymmetry of Aegla platensis Schmitt, 1942 were analyzed. The animals were collected from July 1997 to September 1998 at Arroio do Mineiro, Taquara, Rio Grande do Sul, Brazil ( $\left.29^{\circ} 46^{\prime} \mathrm{S}, 50^{\circ} 53^{\prime} \mathrm{W}\right)$. The sex, length, width of the carapace and the total weight of the crab were registered. And after that, the animals were returned to the stream. In the statistical analysis von Bertalanffy's model was used. The growth curves in length $(\mathrm{mm})$ of males and females are described, respectively, by the following equations: $\mathrm{L}_{\mathrm{t}}=17.39\left[1-\mathrm{e}^{-0.0041(\mathrm{t}+39.13)}\right]$ and $\mathrm{L}_{\mathrm{t}}=19.12\left[1-\mathrm{e}^{-0.0033(\mathrm{t}+}\right.$ 50.38) $]$. The equations that describe the growth in weight $(\mathrm{g})$ of males and females, respectively, are: $\mathrm{Wt}=2.48\left[1-\mathrm{e}^{-0.004(\mathrm{t}+39.13)}\right]^{3.04}$ and $\mathrm{Wt}=3.38\left[1-\mathrm{e}^{-0.0033(\mathrm{t}+}\right.$ $50.38)^{3.05}$. Among $36,5 \%$ males had larger left chelae and $52 \%$ of females had chelae of the same size.

KEY WORDS. Crustacea, Decapoda, Aegla platensis, growth
\end{abstract}

Os crustáceos anomuros do gênero Aegla Leach, 1820 estão presentes em arroios, rios de correnteza e lagos na América do Sul desde o município de Franca, São Paulo, até a ilha Madre de Dios, no Chile (Bond-BuCKUP \& BUCKUP 1994). São elos importantes nas cadeias alimentares dos ambientes límnicos por fazerem parte da dieta de muitos animais como rãs, peixes e aves (ARENAS 1974) e por serem predadores de larvas de simulídeos (MAGNI \& PY-DANIEL 1989).

Um dos primeiros trabalhos sobre a biometria de Aegla, do Uruguai, foi o de VAZ-FERREIRA et al. (1945). Já com respeito ao crescimento e reprodução, um dos pioneiros foi o trabalho de BAHAMONDE \& LOPES (1961) realizado no Chile. LOPEZ (1965) estudou a biologia de Aegla odebrechtti paulensis Schmitt, 1942 (hoje A. paulensis), em São Paulo. RodRIGUES \& HEBLING (1978) estudaram aspectos biológicos e biométricos de Aegla perobae Hebling \& Rodigues, 1977, em São Paulo.

Com relação aos demais crustáceos de água continentais, os camarões são os animais com maior número de informações sobre crescimento. Destacam-se os trabalhos de VALENTI et al. (1987, 1993, 1994), SOUZA \& FonTOURA (1995), BARROS \& FONTOURA (1996) e SAMPAIO \& VALENTI (1996).

1) Contribuição número 304 do Departamento de Zoologia, Universidade Federal do Rio Grande do Sul.

2) Programa de Pós-Graduação em Biologia Animal, Departamento de Zoologia Instituto de Biociências, Universidade Federal do Rio Grande do Sul. Avenida Paulo Gama, prédio 12105, 900040-060 Porto Alegre, Rio Grande do Sul, Brasil. 
Outros trabalhos com crustáceos de águas continentais, tratam sobre o crescimento do lagostim de água doce Parastacus brasiliensis (Von Martens 1869) (FONTOURA 1989), e do caranguejo de marisma Chasmagnathus granulata Dana, 1851 na Lagoa dos Patos, Rio Grande do Sul (D’INCAO et al. 1993).

Com relação à simetria dos quelípodos, destacam-se os trabalhos de LOPEZ (1965) e RODRIGUES \& HEBLING (1978) com aeglídeos, e CARAVELLO \& CAMEROM (1987), GOVIND (1989), MCCLURE (1996) e ROSENBERG (1997) que estudaram a heteroquilia no ocipodídeo Uca Leach, 1814 e em lagostas.

Este trabalho tem como objetivo caracterizar o crescimento de Aegla platensis com o propósito de contribuir para o conhecimento da bio-ecologia dos aeglídeos.

\section{MATERIAL E MÉTODOS}

Exemplares de Aegla platensis Schmitt, 1942 foram coletados de julho/97 a setembro/98, no Arroio do Mineiro, localidade de Fazenda Fialho, município de Taquara, Rio Grande do Sul. O corpo d'água tem aproximadamente $5 \mathrm{~m}$ de largura e uma profundidade média de $30 \mathrm{~cm}$. As coletas foram feitas em uma área com extensão aproximada de $18 \mathrm{~m}$. Em cada saída a campo, os animais foram capturados com auxílio de puçás. No local de coleta, levantou-se todas as pedras mexendo no substrato e na vegetação de uma margem a outra do arroio, sempre no sentido oposto da correnteza, para que tanto jovens e adultos pudessem cair nos puçás. Os crustáceos eram colocados em bacias plásticas, na beira do arroio, para a triagem.

Foram registrados, para cada exemplar, o sexo, o comprimento e a largura do cefalotórax. O sexo dos exemplares foi determinado pela presença ou ausência de pleópodos, que estão presentes somente nas fêmeas. Quando os indivíduos eram muito pequenos, o sexo era identificado pela observação da presença do poro genital feminino na coxa do terceiro pereiópodo. Foram identificados como juvenis os indivíduos menores de $9 \mathrm{~mm}$ de comprimento de carapaça (CC), considerando-se que o comprimento da menor fêmea ovígera foi de $9,87 \mathrm{~mm}$ de CC (BUENO \& BOND-BUCKUP 2000). Para estas medidas, utilizou-se um paquímetro digital Moore \& Wright com precisão de $0,1 \mathrm{~mm}$.

Em cada coleta foram registrados o $\mathrm{pH}$ e a temperatura da água em diferentes pontos do arroio, utilizando um medidor de $\mathrm{pH}$ portátil Hanna e termômetros com escala interna.

Nos meses de novembro de 1997 (primavera), fevereiro de 1998 (verão), maio (outono) e agosto (inverno) todos os animais medidos foram também pesados, utilizando uma balança semi-analítica Fisatom com precisão $0,01 \mathrm{~g}$. Teve-se o cuidado de secar com papel absorvente todos os exemplares e desprezar as fêmeas ovígeras e os indivíduos com apêndices perdidos.

Verificou-se visualmente 0 grau de heteroquilia, ou seja, se havia diferença no tamanho das quelas em todos os machos e fêmeas amostrados. Após a biometria os animais foram devolvidos no mesmo local do arroio em que foram coletados.

\section{Análise estatística}

$\mathrm{O}$ crescimento em comprimento de machos e fêmeas foi estimado, separadamente, através do deslocamento das modas obtidas, em cada mês de amostragem, nas distribuições de freqüências absolutas por intervalo de classe de comprimento 
(MACDONALD \& PITCHER 1979; MACDONALD 1987). As distribuições de freqüências foram obtidas através de programa estatístico (Microstat desenvolvido por Ecosoft, Inc. 1983/1984).

As modas que puderam ser melhor identificadas para o cálculo das curvas de crescimento foram oriundas das coletas realizadas nos meses de outubro, novembro, janeiro, março, julho e setembro para machos e outubro, novembro, dezembro, janeiro, março, junho e setembro para as fêmeas.

Para a descrição do crescimento de machos e fêmeas utilizou-se o modelo de BERTALANFFy (1938): $\mathbf{C}_{\mathbf{t}}=\mathbf{C}_{\infty}\left[\mathbf{1}-\mathrm{e}^{-\mathrm{k}(\mathrm{t}+\mathrm{t} \text { to }}\right]$ sendo: $\left(\mathrm{C}_{\mathrm{t}}\right)$ comprimento médio do cefalotórax dos indivíduos com idade $t$, em milímetros; $\left(\mathrm{C}_{\infty}\right)$ comprimento médio máximo da carapaça, em milímetros; $(\mathrm{k})$ parâmetro relacionado com a taxa de crescimento, referente a dias; (e) base dos logaritmos naturais; (t) idade dos indivíduos, em dias; $\left(\mathrm{t}_{\mathrm{o}}\right)$ parâmetro relacionado com o comprimento do animal ao nascer.

As curvas foram linearizadas pelo método proposto por ALLEN (1976) e as retas foram comparadas utilizando análise da covariância para um intervalo de confiança de 95\%, adotando-se os procedimentos recomendados por SNEDECOR \& COCHRAN (1967).

A relação entre comprimento do cefalotórax e peso, para machos e fêmeas, segundo RICKER (1975), foi descrita através da seguinte equação: $\mathrm{P}=\mathrm{a} C^{\mathrm{b}}$, sendo: (P) peso em gramas; (C) comprimento do cefalotórax em milímetros; (a) fator de condição; (b) parâmetro da relação comprimento/peso.

A determinação da curva de crescimento em peso foi obtida a partir das relações comprimento/ peso e das curvas de crescimento em comprimento, pela seguinte equação: $\mathbf{P}_{\mathbf{t}}=\mathbf{P}_{\infty}\left[1-\mathrm{e}^{-\mathbf{k}\left(\mathbf{t}+\mathbf{t o}_{0}\right)}\right]^{\mathbf{b}}$ sendo: $\left(\mathrm{P}_{\mathrm{t}}\right)$ peso total médio dos indivíduos com idade $t$, em gramas; $\left(\mathrm{P}_{\infty}\right)$ peso total médio máximo que os indivíduos podem atingir, em gramas; (k) parâmetro relacionado com a taxa de crescimento, referente a dias; (e) base dos logaritmos naturais; (t) idade dos indivíduos, em dias; $\left(\mathrm{t}_{\mathrm{o}}\right.$ ) parâmetro relacionado com o comprimento do animal ao nascer; b: parâmetro relacionado com o comprimento e peso.

\section{RESULTADOS E DISCUSSÃO}

As figuras 1 e 2 apresentam, respectivamente, as distribuições de freqüências absolutas mensais do comprimento do cefalotórax (milímetros) de machos e fêmeas. Em ambas as distribuições de freqüências observa-se a maior freqüência de juvenis no mês de outubro. Optou-se, então, por acompanhar o crescimento dos animais a partir deste mês.

As curvas de crescimento em comprimento do cefalotórax de machos e fềmeas são descritas pelas seguintes equações (Figs 3-4):

$\mathrm{C}_{\mathrm{t}}=17,39\left[1-\mathrm{e}^{-0,0041(\mathrm{t}+39,13)}\right] ; \mathrm{n}=1303 ; \mathrm{r}=0,96$ (machos)

$\mathrm{C}_{\mathrm{t}}=19,12\left[1-\mathrm{e}^{-0,0033(\mathrm{t}+50,38)}\right] ; \mathrm{n}=1219 ; \mathrm{r}=0,87$ (fêmeas)

A tabela I mostra o resultado da comparação das curvas de crescimento linearizadas. Como $F_{c}=10,47$ é maior do que $F_{0,05}$, gl 6,5 rejeita-se a hipótese da existência de homogeneidade das variância residuais a nível de $95 \%$. Na comparação da declividade ou coeficiente de regressão, entre machos e fêmeas, encontrou-se um valor de $\mathrm{F}_{\mathrm{c}}<\mathrm{F}_{\alpha}$, concluindo-se que não há diferença significativa entre os valores de $b$. 

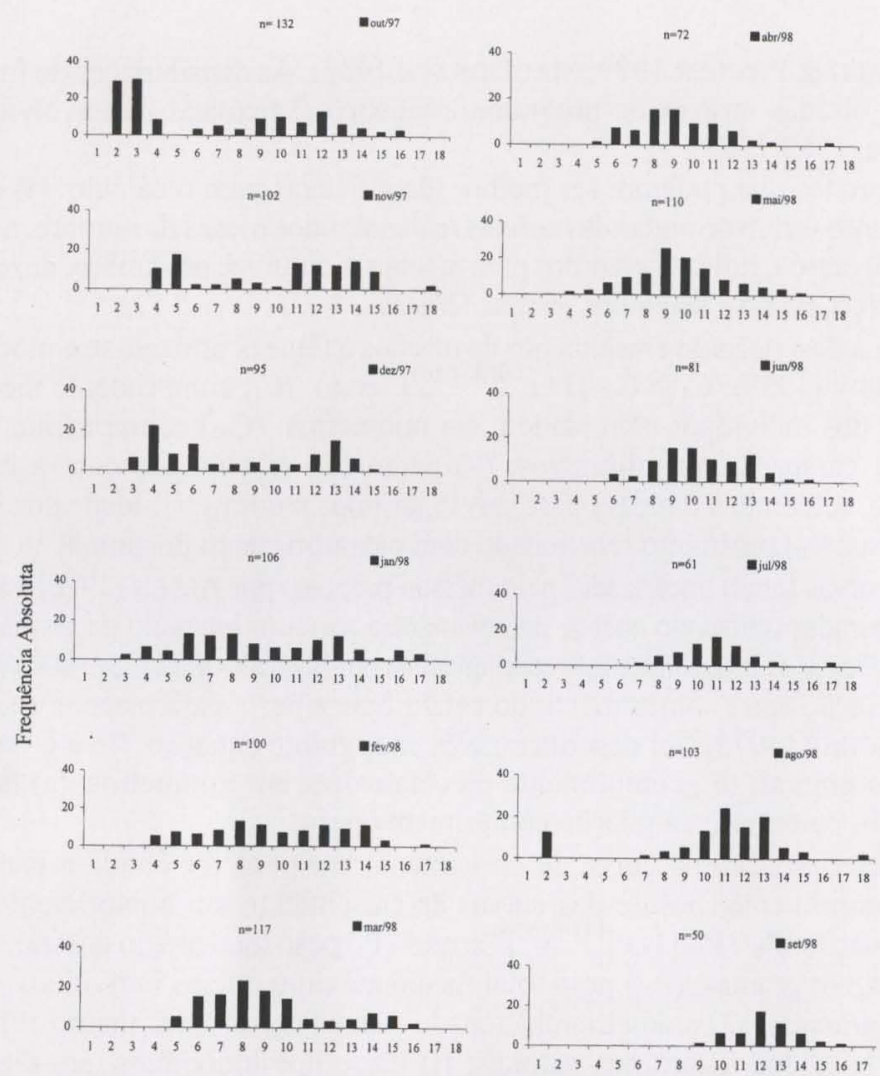

Categorias

Fig. 1. Aegla platensis, machos. Distribuição de freqüências absolutas mensais do comprimento do cefalotórax $(\mathrm{mm})$, de individuos coletados de outubro/97 a setembro/98, no Arroio do Mineiro, município de Taquara, Rio Grande do Sul (Categorias: $1=0$-| 1,2mm; 2=1,2 -| $2,4 \mathrm{~mm} ; 3=2,4-|3,6 \mathrm{~mm} ; 4=3,6-| 4,8 \mathrm{~mm} ; 5=4,8-|6,0 \mathrm{~mm} ; 6=6,00-| 7,2 \mathrm{~mm} ; 7=7,2-\mid 8,4 \mathrm{~mm}$; $8=8,4-|9,6 \mathrm{~mm} ; 9=9,6-| 10,8 \mathrm{~mm} ; 10=10,8-|12,0 ; 11=12,0-| 13,2 \mathrm{~mm} ; 12=13,2-\mid 14,4 \mathrm{~mm}$; $13=14,4-|15,6 \mathrm{~mm} ; 14=15,6-| 16,8 \mathrm{~mm} ; 15=16,8-|18,0 \mathrm{~mm} ; 16=18,0-| 19,2 \mathrm{~mm} ; 17=19,2$ $-|20,4 \mathrm{~mm} ; 18=20,4-| 21,6 \mathrm{~mm})$.

Tabela I. Aegla platensis. Comparação entre as regressões do comprimento ( $\mathrm{mm}$ ) para a idade (dias), para machos e fêmeas coletados no Arroio do Mineiro, município de Taquara, Rio Grande do Sul, durante o periodo de outubro/97 a setembro/98. $F\left(s^{2}\right)$ : Valor de $F$, calculado pela comparação das variâncias residuais; $F(a)$ : valor de $F$, calculado pela comparação dos valores de a; $F(b)$ : valor de $F$, calculado pela comparação dos valores de b.

\begin{tabular}{cccc}
\hline Sexo & $F\left(s^{2}\right)$ & $F(a)$ & $F(b)$ \\
\hline Macho/fêmea & $F(10,47)^{*}$ & $F(3,03) N S$ & $F(3,02) N S$ \\
\hline
\end{tabular}

(*) Significante para $\alpha$ igual ou menor do que 0,05 e maior do que 0,01 ; (NS) não significante para $\alpha$ igual a 0,05 . 

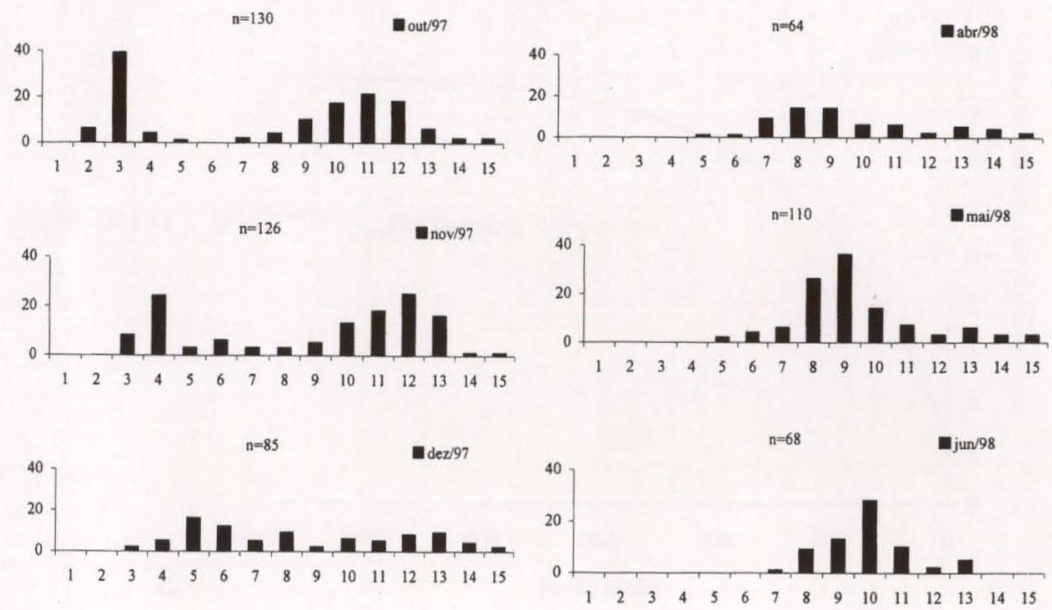

晃
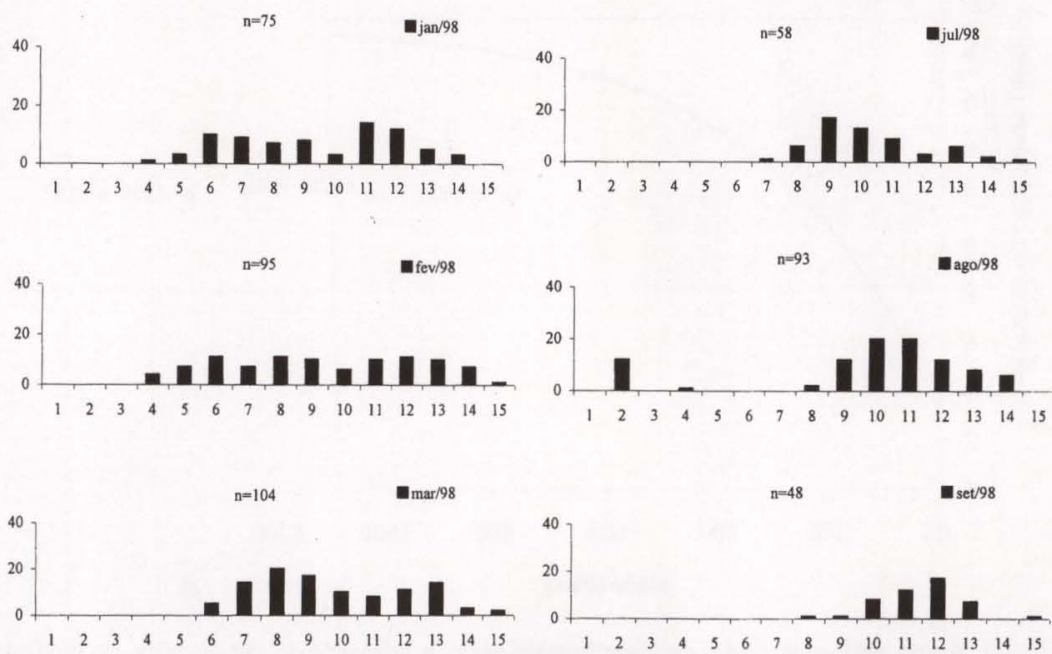

Categorias

Fig. 2. Aegla platensis, fêmeas. Distribuição de freqüências absolutas mensais do comprimento do cefalotórax $(\mathrm{mm})$, de indivíduos coletados de outubro/97 a setembro/98, no Arroio do Mineiro, municipio de Taquara, Rio Grande do Sul (Categorias: $1=0-|1,2 \mathrm{~mm} ; 2=1,2-| 2,4 \mathrm{~mm}$; $3=2,4-|3,6 \mathrm{~mm} ; 4=3,6-| 4,8 \mathrm{~mm} ; 5=4,8-|6,0 \mathrm{~mm} ; 6=6,00-| 7,2 \mathrm{~mm} ; 7=7,2-\mid 8,4 \mathrm{~mm} ; 8=8,4$ $-9,6 \mathrm{~mm} ; 9=9,6-|10,8 \mathrm{~mm} ; 10=10,8-| 12,0 ; 11=12,0-|13,2 \mathrm{~mm} ; 12=13,2-| 14,4 \mathrm{~mm} ; 13=$ $14,4-|15,6 \mathrm{~mm} ; 14=15,6-| 16,8 \mathrm{~mm} ; 15=16,8-\mid 18,0 \mathrm{~mm})$.

O crescimento diferencial dos sexos é bem conhecido entre os crustáceos. BAHAMONDE \& LOPEZ (1961) verificaram diferenças em espécies chilenas de Aegla, sem adotar análise estatística que comprovasse as observações. Em camarões de água doce foi observado crescimento diferencial entre machos e fêmeas (VALENTI et al. 1987; SOUZA \& FONTOURA 1995; BARROS \& FOUNTOURA 1996). 

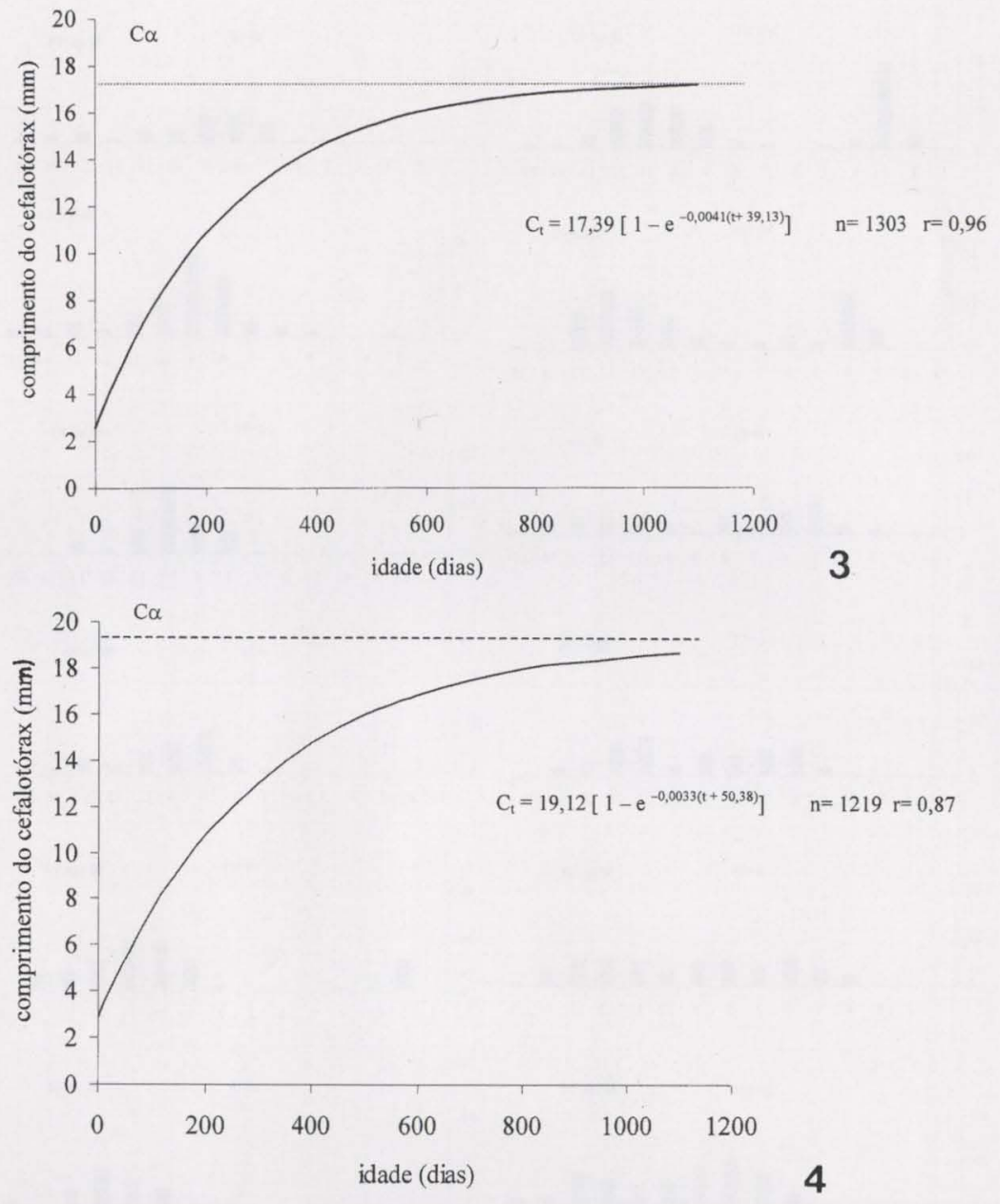

Figs 3-4. Aegla platensis, curva de crescimento em comprimento do cefalotórax, de indivíduos coletados de outubro/97 a setembro/98, no Arroio do Mineiro, município de Taquara, Rio Grande do Sul. (3) Machos; (4) fêmeas. ( $\left.C_{t}\right)$ Comprimento do cefalotórax ( $\mathrm{mm}$ ) dos indivíduos no tempo $t,\left(\mathrm{C}_{\infty}\right)$ comprimento médio máximo, $(\mathrm{t})$ idade em dias.

O comprimento médio máximo das fêmeas $(19,12 \mathrm{~mm})$ foi maior que os dos machos (17,39 mm). Isto ocorreu, não rorque os machos são menores que as fêmeas, mas porque machos com comprimento superior a $17,39 \mathrm{~mm}$ foram raros na população. Esporadicamente, foram capturados machos com até $21 \mathrm{~mm}$ de comprimento. Uma explicação possível seria de que os machos mais velhos migrem para outros pontos do arroio, impossibilitando a sua captura. A propósito, LOPEZ (1965) observou que Aegla odebrechtti paulensis Schmitt, 1942 (hoje A. paulensis) pode deslocar-se por uma distância de até $300 \mathrm{~m}$ ao longo do arroio.

Para cada sexo foi obtida uma relação do comprimento/ peso do cefalotórax, descritas pelas equações que seguem: 


$$
\begin{aligned}
& \mathrm{P}=0,000421 \mathrm{C}^{3,0427} ; \mathrm{n}=398 ; \mathrm{r}=0,89 \text { (machos) } \\
& \mathrm{P}=0,000417 \mathrm{C}^{3,0546} ; \mathrm{n}=388 ; \mathrm{r}=0,91 \text { (fêmeas) }
\end{aligned}
$$

Os valores obtidos revelam a semelhança entre os dois sexos para relação comprimento/peso.

As equações que descrevem o crescimento em peso para machos e fêmeas são descritas abaixo (Figs 5-6):

$$
\begin{aligned}
& \mathrm{P}_{\mathrm{t}}=2,48\left[1-\mathrm{e}^{-0,0041(\mathrm{t}+39,13)}\right]^{3,04} \text { (machos) } \\
& \mathrm{P}_{\mathrm{t}}=3,38\left[1-\mathrm{e}^{-0,0033(\mathrm{t}+50,38)}\right]^{3,05} \text { (fềmeas) }
\end{aligned}
$$

Devido ao fato de que o comprimento médio máximo do cefalotórax das fêmeas foi maior que o dos machos, o peso médio máximo das fêmeas também foi maior.

A adoção da equação de von Bertalanffy mostrou ser apropriada para o estudo do crescimento em comprimento e crescimento em peso de Aegla platensis, com significância de 95\%. Assim como VALENTI et al. $(1987,1993,1994)$ FoNTOURA (1989), D'INCAO et al. (1993), SAMPAIO \& VALENTI (1996) e BARROS \& FONTOURA (1996) também obtiveram sucesso no uso da referida equação na caracterização do crescimento de outros crustáceos.

O grau de heteroquilia para machos foi de $36,5 \%$ para quela esquerda e $43 \%$ apresentaram quelas iguais. Já $51,7 \%$ das fêmeas apresentaram quelas do mesmo tamanho. Estes resultados diferem, em parte, do encontrado por LoPEZ (1965) com Aegla paulensis, onde $72 \%$ dos machos e $82,5 \%$ das fêmeas apresentaram heteroquilia com predomínio da quela esquerda. RoDRIGUES \& HEBLING (1978) encontraram em $86,89 \%$ dos machos e $97 \%$ das fêmeas a quela esquerda maior que a direita na mesma espécie. A diferença no tamanho das quelas pode estar relacionado ao uso diferencial dos pereiópodos durante a fase jovem, onde o sistema nervoso central tem papel fundamental (Govind 1989). No ocipodídeo Uca, observou-se que o quelípodo maior, presente somente nos machos, têm funções de "displays" utilizados durante a corte e em combates, para ataque e/ou defesa. Já o quelípodo menor é usado somente na alimentação (CARAVELlo \& CAMEROM 1987; ROSENBERG 1997). Essas ponderações poderiam explicar porque os machos de $A$. platensis apresentam heteroquilia. Por outro lado, MCCLURE (1996) verificou que ocorre uma inversão no tamanho das quelas quando a maior é perdida. Durante a regeneração da quela maior há um estímulo nervoso que faz com que esta se torne menor mesmo depois de regenerada.

HARTNOLL (1985) menciona que além das limitadas informações sobre crescimento em anomuros, o taxon é muito heterogêneo não permitindo fazer generalizações sobre modelos de crescimento. Entretanto, este grupo pode ser considerado como tendo crescimento indeterminado, ou seja, o animal sofre ecdises contínuas após a puberdade. Nesta perspectiva a espécie estudada, A. platensis, se enquadra perfeitamente.

Finalmente verificou-se que a temperatura da água, do arroio do Mineiro, variou de $13^{\circ} \mathrm{C}$ (setembro/97 e maio/98) a $22^{\circ} \mathrm{C}$ (dezembro/97). O pH permaneceu sempre em torno de 7, variação de 7,03 (fevereiro/98) a 7,98 (março/98), corroborando com os dados obtidos por LOPEZ (1965) que obteve valores semelhantes nos 
habitat de A. paulensis. Aegla platensis vive em águas lóticas, límpidas e de fundo pedregoso, podendo ser encontrados em remansos do corpo d'água onde se acumulam folhas e detritos.
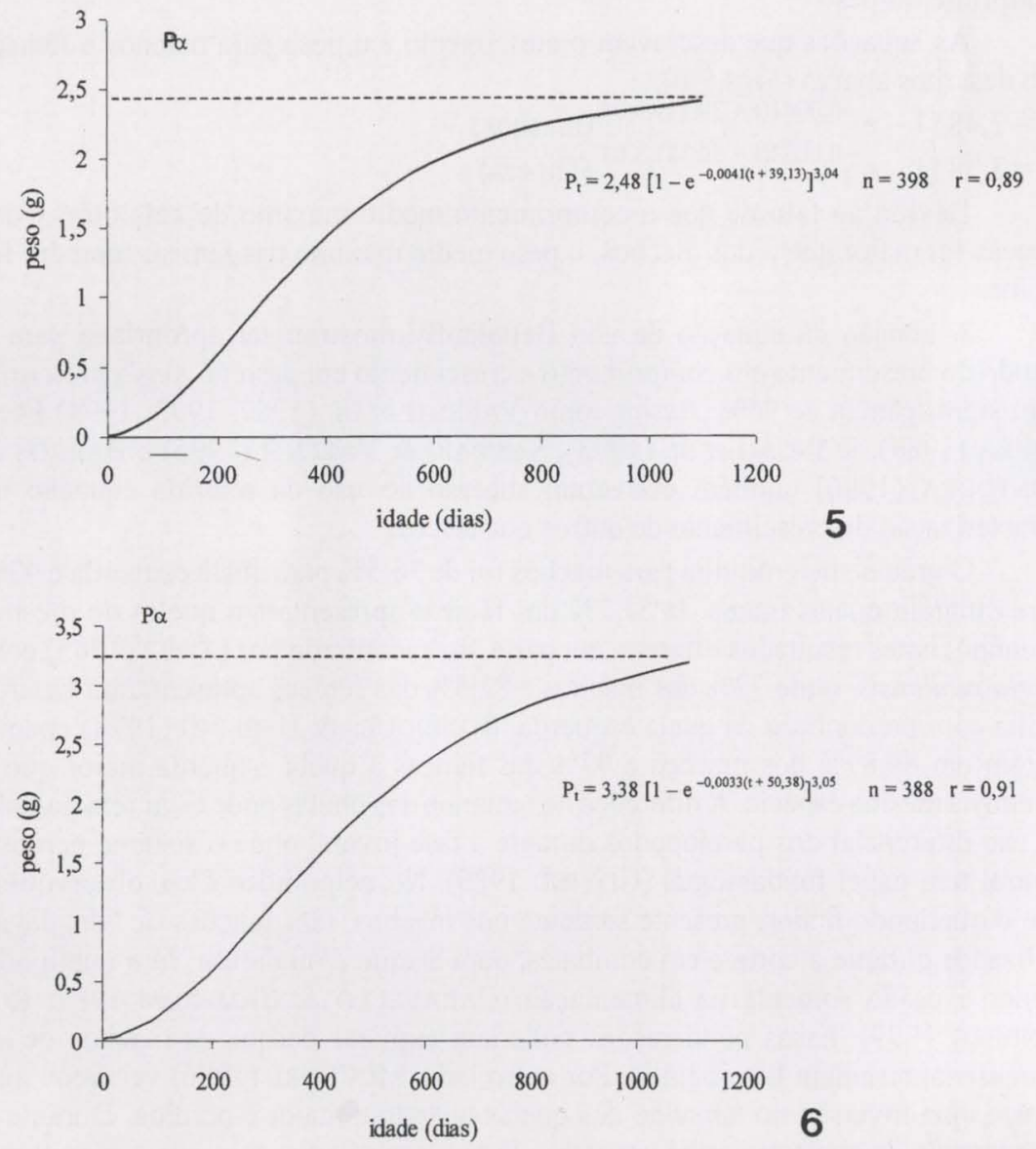

Figs 5-6. Aegla platensis, curva de crescimento em peso, de individuos coletados de outubro/97 a setembro/98, no Arroio do Mineiro, municipio de Taquara, Rio Grande do Sul. (5) Machos; $(6)$ fêmeas. $\left(P_{t}\right)$ Peso $(g)$ dos indivíduos no tempo $t_{1}\left(P_{\infty}\right)$ peso médio máximo e $(t)$ idade em dias.

AGRADECIMENTOS. Ao Dr. Sandro Santos, Dr. Sérgio de Siqueira Bueno e Dra Inga Ludmila Veitenheimer-Mendes que avaliaram a dissertação de mestrado do primeiro autor. À CAPES pela bolsa concedida a nível de mestrado. Ao CNPq pela bolsa de produtividade em pesquisa do segundo autor. Ao Programa de Pós-Graduação em Biologia Animal pelos auxílios concedidos para realização dos trabalhos de campo. 


\section{REFERÊNCIAS BIBLIOGRÁFICAS}

AlLeN, R.L. 1976. Method for comparing fish growth curves. N.Z. Jour. Mar. Freshwat. Res. 10 (4): 687-692.

ARENAS, J. 1974. La cordillera de la costa como refugio de la fauna dulcícola preglacial. Arch. Biol. Med. Exp. 10: 1-40.

Bahamonde, N. \& M.T. Lopez. 1961. Estudios biologicos en la populacion de Aegla laevis laevis (Latreille) de el Monte (Crustacea, Decapoda, Anomura). Investnes. zool. chil. 7: 19-58.

Barros, M.P. \& N.F. Fontoura. 1996. Crescimento de Potimirim glabra (Kingsley 1878) (Crustacea, Decapoda, Atyidae) na Praia da Vigia, Garopaba, Santa Catarina, Brasil. Nauplius 4: 11-28.

BertalanFFy, L. 1938. A quantitative theory of organic growth (inquiries on growth laws II). Hum. Biol. 10 (1): 181-213.

Bond-Buckup, G. \& L. Buckup. 1994. A família Aeglidae (Crustacea, Decapoda, Anomura). Arch. Zool. Est. São Paulo 2 (4): 159-346.

Bueno, A.A.P. \& G. Bond-Buckup. 2000. Dinâmica populacional de Aegla platensis Schmitt, em Ambiente Natural (Crustacea, Decapoda, Aeglidae). Revta bras. Zool. 17 (1): 43-49.

CARAvello, H.E. \& G.N. CAmeron. 1987. The effects of sexual selection on the foranging behavior of the Gulf Coast fiddler crab, Uca panacea. Anim. Behav. 35 (6): 1864-1874.

D'Incao, F.; M.L. Ruffino; K.G. Silva; A.C. Braga \& L.H.C. Marques. 1993. Crescimento de Chasmagnathus granulata Dana, 1851, em um marisma do estuário da Lagoa dos Patos, RS (Decapoda, Grapsidae). Rev. Brasil. Biol. 53 (4): 637-643.

Fontoura, N.F. 1989. Crescimento de Parastacus brasiliensis (Von Martens, 1869) (Crustacea, Decapoda, Parastacidae). Rev. Brasil. Biol. 49 (4): 897-909.

Govind, C.K. 1989. Asymmetry in lobster claws. Amer. Scient. 77: 468-474.

HARTNOLL, R.G. 1985. Growth, sexual maturity and reproductive output. In: A.M. WenNER (Ed.), p. 101-128. Crustacean Issues: Factors in Adult Growth. Rotterdam, A.A. Balkema, Vol. 3, 362p.

LOPEZ, M.T. 1965. Estudios biologicos en Aegla odebrechtti paulensis, Schmitt (Crustacea, Decapoda, Anomura). Bol. Zool. Fac. Fil. Cien. Letras, São Paulo, 25: 301-314.

MacDonald, P.D.M. 1987. The analysis of length-frequency distributions, p.371-384. In: R.C. Summerfelt \& G. Hall. Age and growth of fish. Ames, Iowa State University Press.

MACDonald, P.D.M. \& T.J. PitChER. 1979. Age-groups from size-frequency data: a versatile and efficient method on analyzing distribution mixtures. Jour. Fish. Res. Board. Can. 36: 987-1001.

MaGNI, S.T. \& V. Py-DANIEl. 1989. Aegla platensis Schmitt,1942 (Decapoda, Anomura) um predador de imaturos de Simullidae (Diptera, Culicomorpha). Revta Saúde Públ., São Paulo, 23 (3): 258-259.

MCCluRE, M.R. 1996. Symmetry in large claws of snapping shrimp in nature (Decapoda, Alpheidae). Crustaceana 69 (7): 920-921.

RICKER, W.E. 1975. Computation and interpretation of biological statistics of fish populations. Bull. Fish. Res. Board Can. 191: 1-382.

Rodrigues, W. \& N.J. Hebling. 1978. Estudos biológicos em Aegla perobae Hebling \& Rodrigues, 1977 (Decapoda, Anomura). Rev. Brasil. Biol. 38 (2): 383-390.

RoSENBERG, M.S. 1997. Evolution of shape differences between the major and minor chelipeds of $U c a$ pugnax (Decapoda: Ocypodidae). Jour. Crust. Biol. 17 (1): 52-59.

SAMPAIO, C.M. \& W.C. VALENTI. 1996. Growth for Macrobrachium rosenbergii in semi-intensive culture in Brazil. Jour. Wor. Aquac. Soc. 27 (3): 353-358.

SNEDECOR, C.L. \& W.G. CoCHRAN. 1967. Statistical Method. Ames, The Iowa State University Press, $6^{\mathrm{a}}$ ed., 593p.

SouzA, G.D. \& N.F. Fontoura. 1995. Crescimento de Macrobrachium potiuna no Arroio Sapucaia, município de Gravataí, Rio Grande do Sul (Crustacea, Decapoda, Palaemonidae). Rev. Brasil. Biol. 55 (Supl. 1): 51-63.

VALENTI, W.C.; J.T.C. DE MELlo \& V.L. LoBÃo. 1987. Crescimento de Macrobrachium acanthurus (Wiegmann 1836) do Rio Ribeira de Iguape (Crustacea, Decapoda, Palaemonidae). Rev. Bras. Biol. 47 (3): $349-355$. 
VALENTI, W.C.; J.T.C. DE MELLO \&. N. CASTAGNOLLI. 1993. Efeito da densidade populacional sobre as curvas de crescimento de Macrobrachium rosembergii (de Man) em cultivo semi-intensivo (Crustacea, Palaemonidae). Revta bras. Zool. 10 (3): 371-558.

VALENTI, W.C.; J.T.C. MELLo \&. V.L. LOBÃo. 1994. Maturation and growth curves of Macrobrachium carcinus (Linnaeus) (Crustacea, Decapoda, Palaemonidae) from Ribeira de Iguape River, southern Brazil. Revta bras. Zool. 11 (4): 649-658.

VAz-Ferreira, R.; R. Gary \& M. Vaz-Ferreira. 1945. Notas biométricas sobre los crustáceos decapodos del gênero Aegla Leach, I. La variación de algunas magnitudes en Aegla uruguayana Schmitt. Commun. Zool. Mus. Historia nat. Montevideo 1 (24): 1-6.

Recebido em 31.III.1999; aceito em 01.II.2000. 